\title{
Simultaneous Enterococcus faecalis mitral valve endocarditis, mycotic aortoiliac, and intracranial aneurysms: A surgical dilemma
}

Kei Woldendorp, MD(Dtn), ${ }^{\text {a,b,c,d }}$ Christopher N. Selvaraj, MBBS, ${ }^{\text {a,b }}$ Paul G. Bannon, PhD, FRACS, ${ }^{\text {a,c,de,f }}$ and Steven Dubenec, MBBS, FRACS, ${ }^{\text {a,b }}$ Sydney, Australia

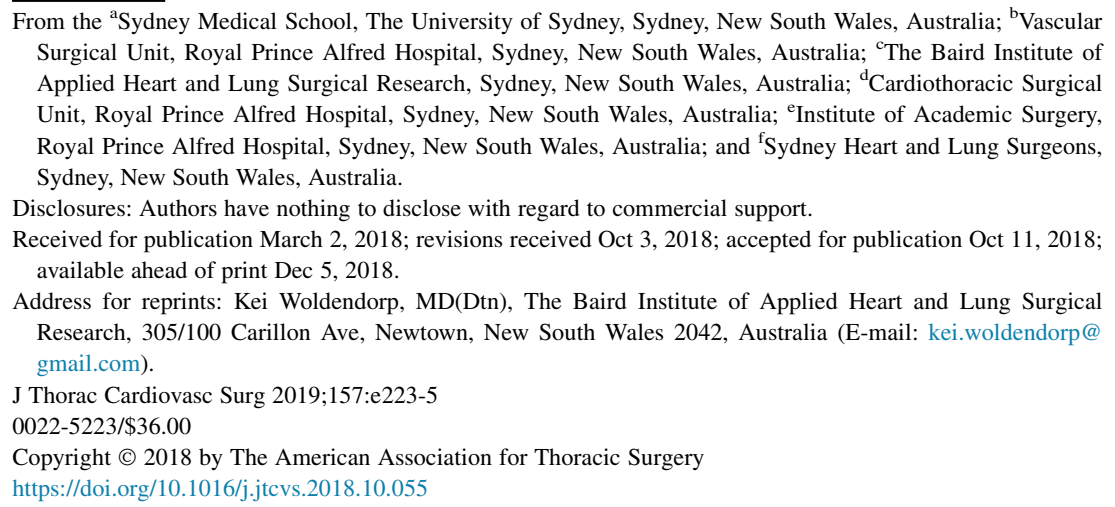

Sir William Osler first described mycotic aneurysm (MA) as a complication of infective endocarditis (IE) in his seminal article in $1885 .{ }^{1}$ Since then, the term has come to include all causes of infective arterial degeneration, but is most commonly associated with transient bacteremia and Salmonella spp. ${ }^{2}$ Mycotic aortic aneurysms (MAAs) comprise $<5 \%$ of all aortic aneurysms, but have a high risk of rupture if not treated urgently. Concomitant valvular IE poses additional therapeutic challenges in relation to staged repair. ${ }^{2-4}$ Here we describe a case of a 60 -year-old male patient presenting with Enterococcus faecalis native mitral valve IE with associated MAA who successfully underwent staged repair.

\section{CLINICAL SUMMARY}

A 63-year-old man (height, $180 \mathrm{~cm}$; weight, $84 \mathrm{~kg}$; body surface area, $2.05 \mathrm{~m}^{2}$ ) was admitted to our facility with moderate-severe mitral regurgitation secondary to mitral valve IE, complicated by multiple systemic septic embolic phenomenon (Figure 1). He initially sought care in another hospital where he presented with symptoms of heart failure and lower limb pain several weeks after a dental treatment. Computed tomography imaging revealed a large saddle embolus at his aortic bifurcation that was removed via bilateral iliofemoral thromboembolectomy followed by systemic heparin anticoagulation therapy. On admission to our facility, he had evidence of expressive dysphasia and left hemianopia. Imaging demonstrated a mycotic aneurysm at the aortic bifurcation and right occipital and left frontoparietal lobe mycotic infarcts. A staged repair approach was taken based on recommendations by a (CPB).

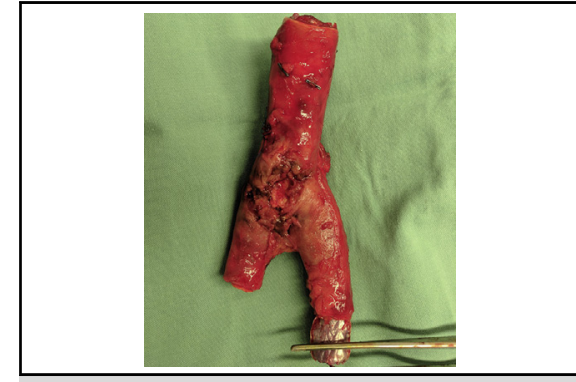

Explanted mycotic aortoiliac bifurcation aneurysm and infected temporizing EVAR graft.

\section{Central Message}

In concomitant infective endocarditis and mycotic aortic aneurysm, a multidisciplinary team can successfully manage the risks of ongoing septic emboli against cardiopulmonary bypass in a friable aorta.

See Commentary on page e227.

multidisciplinary cardiovascular team and to decrease risk of intracranial hemorrhage during cardiopulmonary bypass

To avoid rupture while the patient was being treated with intravenous antibiotics, we elected to temporarily exclude the MAA with an endovascular stent via bilateral femoral artery cutdowns, using a $23 \mathrm{~mm} \times 14.5 \mathrm{~mm} \times 14 \mathrm{~mm}$ excluder Gore-Tex graft (W. L. Gore and Associates, Flagstaff, Ariz). This was placed under digital subtraction angiography guidance as close to the aortic bifurcation as possible to allow for maximal native aortic tissue for later definitive repair. An additional $14.5 \mathrm{~mm} \times 7 \mathrm{~cm}$ graft was deployed into the right limb to exclude the external iliac aneurysm (Figure 1).

Two days later the patient underwent replacement of his mitral valve via standard median sternotomy and CPB, using bicaval right venous cannulation and antegrade cardioplegia. Via a standard Sonnegaard's incision the anterior leaflet was resected, demonstrating a moderate-sized vegetation and leaflet perforations. The posterior leaflet was preserved. A 29-mm Magna Ease bioprosthetic valve (Edwards Lifesciences, Irvine, Calif) was inserted and secured with 

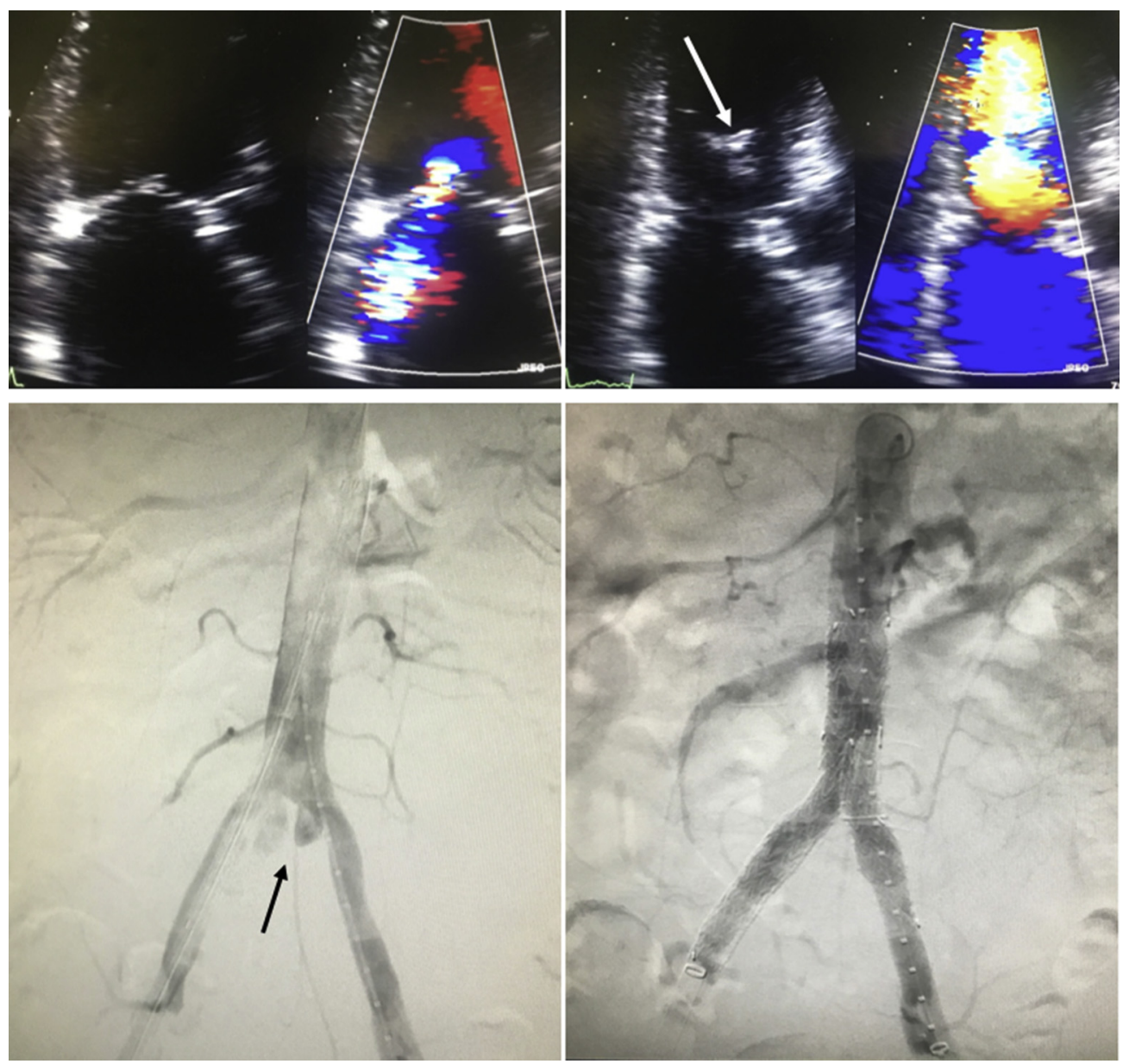

FIGURE 1. Transthoracic echocardiography and angiography findings. Clockwise from top left: moderate-severe mitral regurgitation jet, large anterior mitral valve leaflet vegetation (white arrow), angiogram runs of the abdominal aorta and aortoiliac bifurcation demonstrating the mycotic aneurysm (black arrow) before endovascular temporization, and after successful exclusion of the aneurysm with a bifurcated endovascular aneurysm repair graft.

Cor-Knot (LSI Solutions, Victor, NY). Postoperative transesophageal echocardiography demonstrated a well-seated valve with no regurgitation or perivalvular leak.

The patient was discharged on intravenous ceftriaxone and benzylpenicillin therapy to complete a full 7-week course, per advice from our Infectious Disease Department, before returning for definitive open repair of his MAA via standard midline laparotomy. At operation, an inflammatory phlegmon with surrounding fibrosis was encountered at the distal aortic bifurcation, with no evidence of purulent exudate. The inferior mesenteric and lumbar arteries were oversewn. The infrarenal aorta and bifurcation, along with temporizing endovascular aneurysm repair (EVAR), were excised en bloc and replaced with an $18 \mathrm{~mm} \times 9 \mathrm{~mm}$ bifurcate Gore-Tex graft (Figure 2). Proximal and right distal anastomoses were completed end to end, whereas the left distal anastomosis was completed end to side.
The patient made a full recovery and was discharged home 9 days postlaparotomy on a lifelong course of oral amoxicillin.

\section{DISCUSSION}

Although Enterococcus spp are the third most common pathogens isolated in native valve endocarditis, ${ }^{3}$ the presence of concomitant MAA is rare. Our review found only 3 reports of simultaneous IE and MA, and this is the first involving an Enterococcus spp pathogen.

Current surgical recommendations for MA involve urgent repair due to the high risk of rupture and associated mortality. ${ }^{5}$ The presence of IE in our patient necessitated early valve replacement to avoid further systemic emboli or seeding of vascular graft(s); however, the risk of heparin loading during CBP on a friable aorta was significant.

EVAR provides durable long-term outcomes with significantly reduced morbidity compared with open surgical 

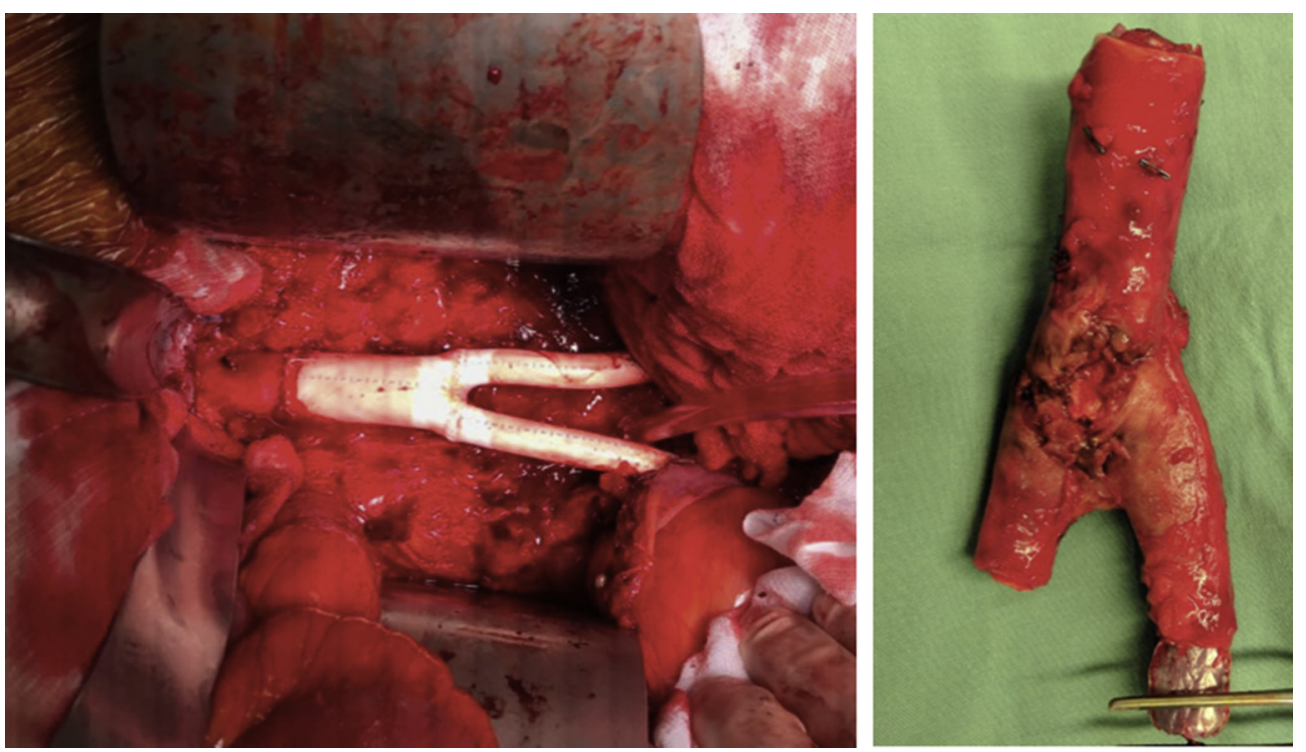

FIGURE 2. Intraoperative findings from definitive repair of mycotic aortoiliac bifurcation aneurysm. Left, Gore-Tex (W. L. Gore and Associates, Flagstaff, Ariz) abdominal bifurcation graft in situ. Right, Mycotic aortoiliac bifurcation aneurysm and infected temporizing endovascular aneurysm repair graft.

options; however, the use in MA remains novel. Case reports and reviews of smaller series examining EVAR in MAA demonstrate early survival benefits but significant long-term rates of graft infection and associated mortality and morbidity. Recently, the American Heart Association recommended EVAR as a bridge to therapy for patients with rupture, unstable hemodynamic parameters, or aortic fistulas, and as a destination therapy for those considered unfit for open surgery. Definitive repair with in situ grafting or extra-anatomic revascularization are the 2 gold-standard surgical options. ${ }^{5}$

\section{References}

1. Osler W. The Gulstonian Lectures, on malignant endocarditis. BMJ. 1885;1:522-6.

2. Airhart N, Curci J, eds. Arterial aneurysms. In: Rutherford's vascular surgery. 8th ed. New York: Elsevier; 2014:113-129.

3. Baddour LM, Wilson WR, Bayer AS, Fowler VG, Tleyjeh IM, Rybak MJ, et al Infective endocarditis in adults: diagnosis, antimicrobial therapy, and management of complications. Circulation. 2015;132:1435-86.

4. González I, Sarriá C, López J, Vilacosta I, Román AS, Olmos C, et al. Symptomatic peripheral mycotic aneurysms due to infective endocarditis: a contemporary profile. Medicine (Abingdon). 2014;93:42-52.

5. Wilson WR, Bower TC, Creager MA, Amin-Hanjani S, O'Gara PT, Lockhart PB, et al. Vascular graft infections, mycotic aneurysms, and endovascular infections: a scientific statement from the American Heart Association. Circulation. 2016;134: e412-60. 